\title{
Differences of the Voice Parameters Between the Population of Different Hearing Tresholds: Findings by Using the Multi-Dimensional Voice Program
}

\author{
Ferit Akil ${ }^{1}$ Umur Yollu² $\cdot$ Ozcan Ozturk ${ }^{1} \cdot$ Murat Yener $^{1}$ \\ ${ }^{1}$ Otorhinolaryngology Department, Istanbul University Cerrahpasa Medical School, Istanbul; \\ ${ }^{2}$ Otorhinolaryngology Clinic, Gumushane Public Hospital, Gumushane, Turkey
}

\begin{abstract}
Objectives. To compare voice parameters in subjects with different hearing level.
Methods. The evaluation consisted of Multi-Dimensional Voice Program (MDVP) and electroglottography. Group 1 consisted of normal hearing subjects which is bilateral average hearing better than 25 decibels $(\mathrm{dB})$ whereas group 2 consisted of patients who have bilateral average hearing between the 25 and $60 \mathrm{~dB}$ and group 3 consisted of patients who have bilateral average hearing between the 60 and $90 \mathrm{~dB}$. The evaluations were performed on males and females separately.

Results. In female subjects, fundamental frequency (F0), absolute jitter, \%jitter and soft phonation index (SPI) were significantly different between the group 1 and group 2. Also, we detected significant difference on maximum phonation time (MPT), fundamental frequency, absolute jitter and \%jitter, and variable F0 (vF0) values between group 1 and group 3. Male subjects demonstrated significant difference between the group 1 and group 2 in MPT, absolute jitter, $\%$ jitter, vF0, and SPI parameters. Between the group 3 and group 1; differences in absolute jitter, \%jitter, shimmer, $\%$ shimmer, vF0, and SPI were also significant.
\end{abstract}

Conclusion. This study concluded that even mild to moderate hearing losses may affect voice patterns in adults and also females and males react differently to hearing loss in some parameters.

Keywords. Voice; Hearing Loss; Adult; Sex

\section{INTRODUCTION}

Human voice with its ability to transform sounds into speech and enable communication is the foremost attribute that distinguish him from other species. Disorders of voice and speech affect the individual negatively both in social and psychological terms by impairing communication. With technological advances and translation of these advances into medicine, significant progresses have been achieved in the diagnosis and treatment of

- Received December 17, 2015

Revised April 27, 2016

Accepted May 27, 2016

- Corresponding author: UmurYollu

Otorhinolaryngology Clinic, Gumushane Public Hospital, Gumushane

Devlet Hastanesi KBB Poliklinigi, Merkez, Gumushane 29000, Turkey

Tel: +90-505-260-6678, Fax: +90-212-414-3000

E-mail: umuryollu@hotmail.com voice and speech disorders.

Several previous studies have demonstrated significant differences in parameters associated with voice characteristics in patients with hearing loss [1]. The major factor affecting voice in the process of speech is the auditory feedback mechanism and that ensures control of the immediate and subsequent components of speech [1,2]. Auditory feedback has also been found to be effective on phonation and respiratory and articulation mechanisms [2,3]. Immediate auditory feedback is critical in the control of suprasegmental components of speech such as fundamental frequency, jitter and quality. For example, fundamental frequency was higher in congenitally deaf patients compared to individuals with normal hearing $[4,5]$.

In this study, we aimed to investigate and compare the changes of some voice parameters in patients with different levels of hearing loss, using the objective methods of analysis.

Copyright (c) 2017 by Korean Society of Otorhinolaryngology-Head and Neck Surgery.

This is an open-access article distributed under the terms of the Creative Commons Attribution Non-Commercial License (http://creativecommons.org/licenses/by-nc/4.0)

which permits unrestricted non-commercial use, distribution, and reproduction in any medium, provided the original work is properly cited. 


\section{MATERIALS AND METHODS}

The study included patients with hearing loss who presented to the Audiology and Phoniatry Unit of the ENT Department of Cerrahpasa Medical Faculty between March and August 2013. Institutional ethics committee (Istanbul University Cerrahpasa Medical School Institutional Review Board; No. IRB00008089) approved the study protocol and all participants gave written informed consent.

Patients who have profound hearing loss with more than 90 decibels $(\mathrm{dB})$ of average hearing threshold in any of the ears were excluded due to possibility of lack of communication. Individuals with hearing loss for less than 6 months, those aged below 18 years and above 60 years (to make an adult homogeneous group), those with reflux, allergic rhinitis, pharyngitis, laryngitis, upper respiratory tract infections, significant laryngeal pathology, smokers, those with previous laryngeal surgery, profound hearing loss and asthma were excluded from the study. For the control group, same exclusion criteria were applied and "to have a normal voice" was not an inclusion criteria strictly wanted. All the criteria were determined to make homogeneous groups.

Total of 50 patients (26 females and 24 males) with hearing loss and 20 normal hearing subjects (10 females, 10 males) were included in the study. Individuals were divided into three groups. Group 1 consisted of normal hearing subjects (bilateral average hearing better than $25 \mathrm{~dB}$ ) as control group. Group 2 consisted of patients who have bilateral mild to moderate hearing loss (between the $25 \mathrm{~dB}$ and $60 \mathrm{~dB}$ ) and group 3 consisted of patients who have bilateral severe to profound hearing loss (between the $60 \mathrm{~dB}$ and $90 \mathrm{~dB}$ ). Groups were designed according to World Health Organization (WHO) classification of hearing loss [6]. Of the female patients, 12 were included to group 2 and 14 were included to group 3. Of the 24 male patients, 11 were included to group 2 and 13 were included to group 3.

Audiometric evaluations were performed in Audiology Section of Otolaryngology Department, Cerrahpasa Medical School. Patients were grouped according to average of pure tone hearing thresholds of air conduction $(500,1,000$, and 2,000 Hz).

Voice analyses of the patients and control subjects were performed by Multi-Dimensional Voice Program (MDVP; Kay Elemetrics, Lincoln Park, NJ, USA) and electroglottography (Elec-

\section{H I G H L I G G H T S}

- Even mild to moderate hearing losses may affect voice patterns in adults.

- Females and males react differently to hearing loss in some parameters.

- Some of the voice parameters may change due to laryngeal instability and decreased neuromuscular activity secondary to impaired auditory feedback mechanism. troglottograph, Kay Elemetrics). All the evaluations were performed in soundproof room that meets the acoustical criteria of living room [7]. The selected sampling frequency was 44,100 Hz for all of the voice recordings. Sound resolution of samples were 16 bits. The subjects wore a headset with a microphone (C444, AKG Acoustics, Vienna, Austria; MDVP, Kay Elemetrics) and the distance between the microphone probe and the mouth was stabilized as $10 \mathrm{~cm}$.

For the maximum phonation time (MPT) test; we want the patient to take deep breathe and persistently pronounce the vowel "a" with a conscious and comfortable pitch and sound intensity for as long as possible. The test was conducted three times for all the patients; result of the longest sound sample was used for the analysis.

The $\mathrm{S} / \mathrm{Z}$ ratio is obtained by timing the longest duration that a patient can sustain the individual phonemes "s" and " $z$." Unlike "s," the voiced "z" requires phonation (i.e., glottic vibration). The best "s" and " $z$ " effort of at least 3 attempts at each is used to obtain the ratio. The $\mathrm{S} / \mathrm{Z}$ ratio takes less than 3 minutes to obtain.

Absolute jitter and absolute shimmer, \%jitter and \%shimmer, fundamental frequency (F0), variable F0 (vF0), soft phonation index (SPI), harmonics to noise ratio (HNR), S/Z ratio and MPT were recorded by MDVP. Also closed quotient was provided by electroglottographic evaluation.

Statistical analysis was made with IBM SPSS ver. 19 (IBM Co., Armonk, NY, USA). Due to abnormal distribution of the datas detected by descriptive analyses and Ploth Test $(P=0.000$, Kolmogorov Smimov; $P=0.000$, Shapiro Wilk), nonparametric Mann-Whitney $U$-test was used for statistical measurements. $P$ value below 0.05 was considered statistically significant.

\section{RESULTS}

\section{Female group}

For the female group; age, the degree of hearing loss at each ear, MPT, S/Z ratio, F0, absolute jitter and absolute shimmer, \%jitter and $\%$ shimmer, vF0, SPI, HNR and closed quotient results were provided as average values in Table 1.

F0, absolute jitter, \%jitter, vF0, and SPI were detected significantly lower in group 1 than group 2 . With the statistical evaluation between group 1 and group 3, significant differences were observed for MPT, F0, absolute and \%jitter, and vF0 values. MPT was significantly lower while F0, absolute jitter, \%jitter and vF0 were higher in group 3. Parameters were undifferent according to statistical evaluation between group 2 and group 3 .

\section{Male group}

For the male group, age, the degree of hearing loss at each ear, MPT, S/Z ratio, F0, absolute jitter and absolute shimmer, \%jitter and \%shimmer, vF0, SPI, HNR, closed quotient results were provided as average values in Table 2 . 
Table 1. Comparison of the results of female group

\begin{tabular}{lccccccc}
\hline Variable & Group 1 & Group 2 & Group 3 & $P$-value & $P$-value & $P$-value & - \\
\hline Total & 10 & 12 & 14 & - & - & - & \\
Age (yr) & $47 \pm 1$ & $45 \pm 9$ & $49 \pm 6$ & 0.282 & 0.384 & 0.302 & 0.276 \\
Hearing thresold & & & & & & \\
$\quad$ Right & $11.3 \pm 2.8$ & $42.9 \pm 7.6$ & $65.8 \pm 6.3$ & $0.000^{*}$ & $0.000^{*}$ & $0.000^{*}$ & $0.000^{*}$ \\
$\quad$ Left & $11.5 \pm 2.4$ & $40.0 \pm 7.0$ & $65.6 \pm 6.3$ & $0.000^{*}$ & $0.000^{*}$ & $0.000^{*}$ & $0.000^{*}$ \\
S/Z ratio) & $0.94 \pm 0.19$ & $0.95 \pm 0.11$ & $0.88 \pm 0.08$ & 0.254 & 0.700 & 0.345 & 0.060 \\
MPT (sec) & $18.4 \pm 4.5$ & $17.0 \pm 6.0$ & $12.3 \pm 4.0$ & $0.043^{*}$ & 0.300 & $0.001^{*}$ & 0.140 \\
FO (Hz) & $244 \pm 22$ & $252 \pm 29$ & $254 \pm 31$ & $0.048^{*}$ & $0.004^{*}$ & $0.034^{*}$ & 0.120 \\
Value of jitter & $2.9 \pm 1.5$ & $4.0 \pm 1.7$ & $8.4 \pm 1.6$ & $0.023^{*}$ & $0.000^{*}$ & $0.002^{*}$ & 0.527 \\
\%Jitter & $0.7 \pm 0.4$ & $1.7 \pm 0.9$ & $1.2 \pm 0.6$ & $0.032^{*}$ & $0.007^{*}$ & $0.001^{*}$ & 0.800 \\
Value of shimmer & $0.25 \pm 0.1$ & $0.28 \pm 0.1$ & $0.26 \pm 0.1$ & 0.954 & 0.960 & 1.000 & 0.920 \\
\%Shimmer & $2.9 \pm 1.1$ & $3.2 \pm 1.1$ & $3.2 \pm 1.1$ & 0.945 & 0.910 & 1.000 & 0.980 \\
vFO (Hz) & $1.2 \pm 0.4$ & $1.9 \pm 1.2$ & $1.7 \pm 0.4$ & $0.039^{*}$ & $0.032^{*}$ & $0.002^{*}$ & 0.786 \\
HNR (dB) & $0.316 \pm 0.04$ & $0.123 \pm 0.03$ & $0.129 \pm 0.02$ & 0.675 & 0.840 & 0.570 & 0.632 \\
SPI & $2 \pm 1.1$ & $3 \pm 1.4$ & $2.5 \pm 1.1$ & $0.043^{*}$ & $0.038^{*}$ & 0.215 & 0.321 \\
Closed quotient & $47 \pm 6$ & $47 \pm 8$ & $46 \pm 6$ & 0.457 & 0.845 & 0.517 & 0.356 \\
\hline
\end{tabular}

Values are presented as number or mean \pm standard deviation.

MPT, maximum phonation time; FO, fundamental frequency; vFO, variable FO; HNR, harmonics to noise ratio; SPI, soft phonation index.

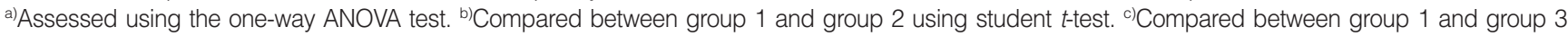
groups student $t$-test. ${ }^{\text {d) }}$ Compared between group 2 and group 3 using student $t$-test. e)Longest duration is recorded that a patient can sustain the individual phonemes "s" and "z." The best "s" and "z" effort of at least 3 attempts at each is used to obtain the ratio.

${ }^{*} P<0.05$.

Table 2. Comparison of the results of male group

\begin{tabular}{|c|c|c|c|c|c|c|c|}
\hline Variable & Group 1 & Group 2 & Group 3 & $P$-value a) & $P$-value & $P$-value ${ }^{c)}$ & $P$-value ${ }^{\text {d) }}$ \\
\hline Total & 10 & 11 & 13 & - & - & - & - \\
\hline Age (yr) & $46 \pm 13$ & $33 \pm 14$ & $44 \pm 12$ & 0.132 & 0.064 & 0.213 & 0.091 \\
\hline \multicolumn{8}{|l|}{ Hearing thresold } \\
\hline Right & $12 \pm 3$ & $43 \pm 8$ & $66 \pm 10$ & $0.000^{*}$ & 0.000 & $0.000^{*}$ & $0.000^{*}$ \\
\hline Left & $13 \pm 3$ & $44 \pm 10$ & $65 \pm 7$ & $0.000^{*}$ & $0.000^{*}$ & $0.000^{*}$ & $0.000^{*}$ \\
\hline $\mathrm{S} / \mathrm{Z}$ ratioe) & $1.07 \pm 0.17$ & $0.97 \pm 0.12$ & $0.96 \pm 0.19$ & 0.267 & 0.212 & 0.274 & 0.765 \\
\hline MPT (sec) & $19.6 \pm 1.7$ & $15.0 \pm 4.4$ & $19.3 \pm 4.2$ & $0.032^{*}$ & $0.005^{\star}$ & 1.000 & $0.037^{\star}$ \\
\hline $\mathrm{FO}(\mathrm{Hz})$ & $196 \pm 1$ & $139 \pm 17$ & $144 \pm 31$ & 0.376 & 0.110 & 0.386 & 0.451 \\
\hline Value of jitter & $2.3 \pm 0.4$ & $7.8 \pm 0.5$ & $7.7 \pm 0.3$ & $0.014^{*}$ & $0.000^{*}$ & $0.000^{*}$ & 0.603 \\
\hline \%Jitter & $0.47 \pm 0.1$ & $0.9 \pm 0.3$ & $1.1 \pm 0.5$ & $0.021^{*}$ & $0.000^{*}$ & $0.003^{*}$ & 0.632 \\
\hline Value of shimmer & $0.30 \pm 0.05$ & $0.34 \pm 0.19$ & $0.42 \pm 0.12$ & $0.470^{*}$ & 0.980 & $0.018^{*}$ & 0.174 \\
\hline \%Shimmer & $3.4 \pm 0.6$ & $3.5 \pm 1.9$ & $3.7 \pm 1.2$ & 0.038 & 0.578 & $0.011^{*}$ & 0.392 \\
\hline $\mathrm{vFO}(\mathrm{Hz})$ & $3.4 \pm 0.6$ & $4.9 \pm 0.9$ & $5.1 \pm 1.1$ & $0.019^{*}$ & $0.007^{\star}$ & $0.009^{*}$ & 0.952 \\
\hline HNR (dB) & $0.117 \pm 0.02$ & $0.135 \pm 0.02$ & $0.117 \pm 0.02$ & 0.432 & 0.059 & 0.741 & 0.067 \\
\hline SPI & $10 \pm 4$ & $29 \pm 15$ & $31 \pm 12$ & $0.028^{*}$ & $0.000^{\star}$ & $0.000^{*}$ & 0.512 \\
\hline Closed quotient & $40 \pm 3$ & $41 \pm 12$ & $41 \pm 7$ & 0.419 & 0.311 & 0.786 & 0.543 \\
\hline
\end{tabular}

Values are presented as number or mean \pm standard deviation.

MPT, maximum phonation time; FO, fundamental frequency; vFO, variable FO; HNR, harmonic noise ratio; SPI, soft phonation index.

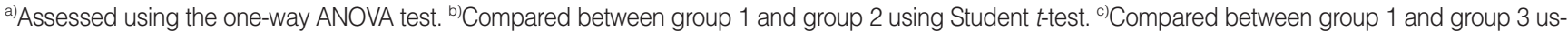
ing Student $t$-test. ${ }^{d)}$ Compared between group 2 and group 3 using Student $t$-test. e) Longest duration is recorded that a patient can sustain the individual phonemes "s" and "z." The best "s" and "z" effort of at least 3 attempts at each is used to obtain the ratio.

${ }^{*} P<0.05$.

Statistical evaluation between the group 1 and group 2 demonstrated significant differences in MPT, absolute jitter and \% jitter, vF0 and SPI. MPT values were significantly higher in group 1 while absolute jitter and \%jitter, $\mathrm{vF0}$, SPI were higher in group 2.
Significant differences were also detected in absolute jitter, \%jitter, shimmer, and \%shimmer, vF0 and SPI according the statistical evaluation between group 1 and group 3. Absolute jitter and \%jitter, absolute shimmer and \%shimmer, vF0 and SPI were significantly higher in group 3. 


\section{DISCUSSION}

Auditory feedback mechanism is the most important factor affecting voice during speech [1] and ensures control of the immediate and subsequent components of speech [2]. Immediate auditory feedback is critical in the control of suprasegmental components of speech such as fundamental frequency, jitter, and quality. Auditory feedback has been found to be effective also on respiratory and articulation mechanisms $[2,3]$.

Many studies in the literature revealed significant differences in parameters associated with voice characteristics in patients with hearing loss when compared to individuals with normal hearing [1]. In several previous studies, fundamental frequency was found to be higher in patients with hearing loss and patients with congenital deafness $[4,5,8]$. People with hearing loss raise the fundamental frequency of their voices so that they may better identify their own voices and thus enhance their perception [9]. However, individual comparisons of patients with hearing loss versus controls yielded significantly higher fundamental frequency values for patients with hearing losses both below and above $60 \mathrm{~dB}$. Our results about this issue was consistent with the literature [8].

In our series, a significant difference was noted in fundamental frequency values for females but not for males, which we consider this as a notable finding. This finding in male patients may be because patients with profound hearing loss were included in many of the previous studies $[3,4]$ while patients with profound hearing loss were not included in ours. It can be notable that our study provide a contribution to Ubrig et al. [10]'s results with the showing of fundemantal frequency changes in also mild and moderate hearing loss patients.

Patients in all groups, males and females, were comparable with regards to age. This indicates that changes in voice, which may occur with age, was not an important factor in our study.

MPT prolongation should be considered in adductor spasmodic dysphonias where glottic closure is jittered, whereas shortening should suggest glottic insufficiency, submaximal effort and pulmonary insufficiency [11]. According to our results; MPT was not different in patients with lower or higher degree of hearing loss but statistically different between patients with higher degree of hearing loss and controls in female patients. In male patients; MPT was significantly higher in patients with higher hearing loss and controls than lower degree of hearing loss. We believe that this disagreement between the results is due to the limited size of the sample. Also one another study has reported that MPT could range from 6.6 to 69.6 seconds [12] and the values in our study were within this range for all patients. It's a controversial issue whether these findings preclude establishing a meaningful relationship between hearing loss and MPT.

$\mathrm{S} / \mathrm{Z}$ ratio allows assessment of the degree of glottic closure and pulmonary functions. A normal S/Z ratio should be $\leq 1.2$
$[13,14]$. S/Z ratios of the patients included in our study were not above 1.2 except for one male patient. In addition, no significant difference was found in terms of $S / Z$ ratio between any of the group pairs. Hence, a significant relationship could not be established between $S / Z$ ratio and hearing loss. Despite there is a reported literature about hearing loss can effect the $S / Z$ ratio [15], this is not compatible with our results.

Jitter refers to the variation in each period. With respect to jitter and \%jitter, the groups with hearing loss below and above $60 \mathrm{~dB}$ in either sex did not differ significantly. There was, however, a significant difference between the groups of patients with hearing loss and the controls, with the former having significantly higher values. This suggests that all degrees of hearing loss may affect jitter. According to previous reports, patients with profound hearing loss have significantly higher jitter values than poeple with normal hearing [9]. Dehqan and Scherer [9] described in their study that increased jitter was due to worsened neuromotor control in patients with hearing loss. In our study, patients with even mild and moderate-jammer hearing loss had significantly higher values compared to controls. We believe that this finding adds value to our study.

Shimmer is expressed as $\mathrm{dB}$ or \% amplitude variation at each glottic cycle. Absolute shimmer and \%shimmer values of the patients in our study were evaluated and a significant difference was observed only between the patients in the male group with hearing loss above $60 \mathrm{~dB}$ and controls. Male patients with hearing loss above $60 \mathrm{~dB}$ had significantly higher values and this result was consistent with the literature [9]. Inconsistency in female results between our study and previous studies may be because our study was performed with lower hearing loss values. Increased shimmer values are also considered an indicator of poor neuromotor control [12] and indicate the negative effect of hearing loss on voice control. According to our study results there is an association between hearing loss and shimmer in males.

Fundamental frequency variability ( $\mathrm{vF} 0$ ) reflects short-term fundamental frequency alternations. Comparison between groups of male and female patients with hearing loss versus controls in our study demonstrated significantly higher $\mathrm{vF} 0$ values in the former. Similarly, higher vF0 values have been reported previously for patients with hearing loss [16]. This was described to be secondary to increased laryngeal instability due to weakened audio-vocal reflex [16]. Our study is of relevance because the data substantiates previous reports in this regard.

HNR is correlated with dysphonia and also used as a sensitive indicator of the vocal function $[11,17,18]$. It is assumed that sound quality will drop as this ratio decreases. Several previous studies have observed decreased HNRs in patients with hearing loss $[9,19]$. In our study, we noted no decreases in HNR that could be associated with different levels of hearing loss. In this study, no statistically significant intergroup differences were noted in HNR parameters between males and females.

SPI $(70-1,600 \mathrm{~Hz})$ is the ratio of mean harmonic energy at 
lower frequencies $(1,600-4,500 \mathrm{~Hz})$ to mean high-frequency harmonic energy. SPI is a parameter that indicates how tense the vocal cords are during phonation, or whether closure is complete. SPI has been found to be significantly higher in patients with hearing loss [20]. The comparison in our study between the group of patients hearing loss below $60 \mathrm{~dB}$ and the control group among females demonstrated significantly higher SPI values in the former, while no significant differences were noted between other groups among females. In males, SPI values were higher in patients with hearing loss both below and above $60 \mathrm{~dB}$ compared to controls. Based on these data, we believe that our study confirms the findings of previous studies with regards to SPI.

With regards to electroglottographic parameters, no significant differences were noted between any of the patient groups and controls in closed quotient. These results indicate that a meaningful correlation can not be made between this parameter and hearing loss.

Mean values for closed quotient were below $50 \%$ for both males and females. The normal range has also been reported to be below $50 \%$ [21]. This suggests that these parameters did not result in pathological differences compared to both the control group in our study and previous studies.

In conclusion, F0, absolute jitter and \%jitter, absolute shimmer and \%shimmer, vF0, SPI may increase due to laryngeal instability and decreased neuromuscular activity secondary to impaired auditory feedback mechanism associated with hearing loss. Although HNR is affected in patients with profound hearing loss, it may not be affected in less severe hearing losses as seen in this study.

This study concluded that even mild to moderate hearing losses may affect voice patterns in adults and also females and males react differently to hearing loss in some parameters. We believe these parameters will take more attention especially in this mild to moderate hearing loss patient group and further studies will focus on this issue carefully. Our study results pointed out that the hearing level and some of the voice parameters are more strictly related than we thought. The major weakness of this study was the small patient population. There is no doubt for that the larger study groups needed to purchase more certain judgments.

\section{CONFLICT OF INTEREST}

No potential conflict of interest relevant to this article was reported.

\section{REFERENCES}

1. Lejska M.Voice field measurements: a new method of examination: the influence of hearing on the human voice. J Voice. 2004 Jun;18 (2):209-15.

2. Yates AJ. Delayed auditory feedback. Psychol Bull. 1963 May;60: 213-32.

3. Siegel GM, Pick HL Jr. Auditory feedback in the regulation of voice. JAcoust Soc Am. 1974 Nov;56(5):1618-24.

4. Angelocci AA, Kopp GA, Holbrook A. The vowel formants of deaf and normal-hearing eleven- to fourteen-year-old boys. J Speech Hear Disord. 1964 May;29:156-60.

5. Giusti MC, Padovani MM, Behlau M, Granato L. The voice of hearing impaired children. Braz J Otorhinolaryngol. 2001 Jan-Feb;67(1): 29-35.

6. Mathers C, Smith A, Concha M. Global burden of hearing loss in the year 2000. Geneva:World Health Organization; 2000.

7. Schutte HK, SeidnerW. Recommendation by the Union of European Phoniatricians (UEP): standardizing voice area measurement/phonetography. Folia Phoniatr (Basel). 1983;35(6):286-8.

8. Leder SB, Spitzer JB, Kirchner JC. Speaking fundamental frequency of postlingually profoundly deaf adult men. Ann Otol Rhinol Laryngol. 1987 May-Jun;96(3 Pt 1):322-4.

9. Dehqan A, Scherer RC. Objective voice analysis of boys with profound hearing loss. JVoice. 2011 Mar;25(2):e61-5.

10. Ubrig MT, Goffi-Gomez MV, Weber R, Menezes MH, Nemr NK, Tsuji DH, et al. Voice analysis of postlingually deaf adults pre- and postcochlear implantation. JVoice. 2011 Nov;25(6):692-9.

11. Rhys Evans PH, Stafford N,Waldron J. Surgical and pathophysiological considerations. In: Singh W, Soutar DS, editors. Functional surgery of the larynx and pharynx. Oxford: Butterworth-Heinemann; 1993. p. 3-17.

12. Baken RJ, Orlikoff RF. Clinical measurement of speech and voice. 2nd ed. San Diego: Singular Publishing Group; 2000.

13. Yuceturk AV, Gunhan K. Multidimensional assessment of voice and speech after supracricoid laryngectomy with cricohyoidopexy. J Laryngol Otol. 2004 Oct;118(10):791-5.

14. Dejonckere PH. Perceptual and laboratory assessment of dysphonia. Otolaryngol Clin North Am. 2000 Aug;33(4):731-50.

15. Selleck MA, Sataloff RT. The impact of the auditory system on phonation: a review. JVoice. 2014 Nov;28(6):688-93.

16. Lee GS. Variability in voice fundamental frequency of sustained vowels in speakers with sensorineural hearing loss. J Voice. 2012 Jan;26(1):24-9.

17. Silver CE. Laryngeal cancer. New York: Thieme Medical Publishers; 1991.

18. Ferrand CT. Harmonics-to-noise ratio: an index of vocal aging. J Voice. 2002 Dec;16(4):480-7.

19. Bench RJ. Communication skills in hearing-impaired children. London:Whurr Publishers; 1992.

20. Mora R, Crippa B, Cervoni E, Santomauro V, Guastini L. Acoustic features of voice in patients with severe hearing loss. J Otolaryngol Head Neck Surg. 2012 Feb;41(1):8-13.

21. Orlikoff RF. Assessment of the dynamics of vocal fold contact from the electroglottogram: data from normal male subjects. J Speech Hear Res. 1991 Oct;34(5):1066-72. 